\title{
SOME QUESTIONS ABOUT ROTUNDITY AND RENORMINGS IN BANACH SPACES
}

\author{
A. AIZPURU ${ }^{\varpi}$ and F. J. GARCIA-PACHECO
}

(Received 31 October 2003; revised 22 September 2004)

Communicated by A. Pryde

\begin{abstract}
In this paper, we show some results involving classical geometric concepts. For example, we characterize rotundity and Efimov-Stechkin property by mean of faces of the unit ball. Also, we prove that every almost locally uniformly rotund Banach space is locally uniformly rotund if its norm is Fréchet differentiable. Finally, we also provide some theorems in which we characterize the (strongly) exposed points of the unit ball using renormings.
\end{abstract}

2000 Mathematics subject classification: primary 46B20.

\section{Introduction}

For a subset $C$ of a bounded closed convex subset $H$ of a $\mathrm{T}_{2}$ topological vector space $X$, it is well known that $C$ is

(i) a face of $H$ if it is closed convex and for every $x, y \in H$ and every $\alpha \in(0,1)$ such that $\alpha x+(1-\alpha) y \in C$, then $x, y \in C$;

(ii) an exposed face of $H$ if there exists $f$ in $X^{*}$ such that $C=\{x \in H: f(x)=$ $\sup (f(H))\}$;

(iii) a strongly exposed face of $H$ if there exists $f$ in $X^{*}$ satisfying $C=\{x \in H$ : $f(x)=\sup (f(H))\}$ and for every open subset $U$ of $H$ with $C \subseteq U$, there exists $\delta>0$ such that $\operatorname{slc}(H, f, \delta) \subseteq U$ (where $\operatorname{slc}(H, f, \delta)=\{h \in H: f(h) \geq \sup (f(H))-\delta\}$ is the slice of $H$ determined by $f$ and $\delta$ ).

If $c$ is an element of $H$, then $c$ is

(i) an extreme point (EXT) of $H$ if $\{c\}$ is a face of $H$;

(C) 2005 Australian Mathematical Society $1446-7887 / 05 \$ A 2.00+0.00$ 
(ii) an exposed point (EXP) of $H$ if $\{c\}$ is an exposed face of $H$;

(iii) a strongly exposed point (SEXP) of $H$ if $\{c\}$ is a strongly exposed face of $H$.

It is not difficult to check that $c$ is

(i) an EXT of $H$ if and only if $H \backslash\{c\}$ is convex;

(ii) an EXP of $H$ if and only if there exists $f$ in $X^{*}$ satisfying $f(y)<f(c)$ for every $y \in H \backslash\{c\}$;

(iii) an SEXP of $H$ if and only if there exists $f$ in $X^{*}$ such that for every sequence $\left(y_{n}\right)_{n \in \mathbb{N}}$ in $H$ with $\left(f\left(y_{n}\right)\right)_{n \in \mathbb{N}}$ convergent to $\sup (H),\left(y_{n}\right)_{n \in \mathbb{N}}$ converges to $c$.

It is well known (see [2-4]) that a point $x$ of the unit sphere of a Banach space $X$ is

(i) a rotund point $(\mathrm{R})$ of $\mathrm{B}_{X}$ if every $y$ in $\mathrm{S}_{X}$, such that $\|(x+y) / 2\|=1$, satisfies $x=y$;

(ii) a midpoint locally uniformly rotund point (MLUR) of $\mathrm{B}_{X}$ if for every pair of sequences $\left(x_{n}\right)_{n \in \mathbb{N}}$ and $\left(y_{n}\right)_{n \in \mathbb{N}}$ in $S_{X}$ such that $\left(\left(x_{n}+y_{n}\right) / 2\right)_{n \in \mathbb{N}}$ converges to $x$, both $\left(x_{n}\right)_{n \in \mathbb{N}}$ and $\left(y_{n}\right)_{n \in \mathbb{N}}$ converge to $x$;

(iii) an SEXP of $B_{X}$ if it is an SEXP of $B_{X}$ for the vector topology given by the norm of $X$;

(iv) an almost locally uniformly rotund point (ALUR) of $\mathrm{B}_{X}$ if for every pair of sequences $\left(x_{n}\right)_{n \in \mathbb{N}}$ in $S_{X}$ and $\left(f_{m}\right)_{m \in \mathbb{N}}$ in $S_{X^{*}}$ such that $\lim _{m}\left(\lim _{n}\left(f_{m}\left(\left(x_{n}+x\right) / 2\right)\right)\right)=1$, $\left(x_{n}\right)_{n \in \mathbb{N}}$ converges to $x$;

(v) a locally uniformly rotund point (LUR) of $\mathrm{B}_{X}$ if every sequence $\left(y_{n}\right)_{n \in \mathbb{N}}$ in $\mathrm{S}_{X}$ such that $\left(\left\|\left(x+y_{n}\right) / 2\right\|\right)_{n \in \mathbb{N}}$ converges to 1 , converges to $x$.

Here we have the following diagram of implications:

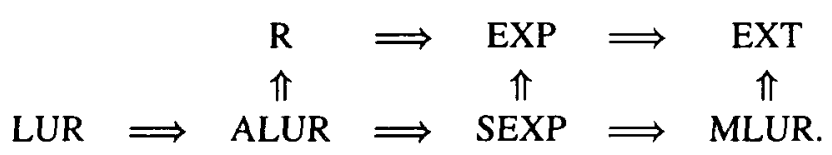

It is said that a Banach space is respectively R, MLUR, SEXP, ALUR or LUR if every point of its unit sphere is an R, MLUR, SEXP, ALUR or LUR point of its unit ball.

It is well known (see [5, Chapter 5.3]) that a point $x$ of the unit sphere of a Banach space $X$ is

(i) a smooth point of $\mathrm{B}_{X}$ if every sequence $\left(f_{n}\right)_{n \in \mathbb{N}}$ in $\mathrm{S}_{X^{*}}$ such that $\left(f_{n}(x)\right)_{n \in \mathbb{N}}$ converges to 1 , verifies that $\left(f_{n}\right)_{n \in \mathbb{N}}$ is $\omega^{*}$-convergent;

(ii) a strongly smooth point of $B_{X}$ if every sequence $\left(f_{n}\right)_{n \in \mathbb{N}}$ in $S_{X}$. such that $\left(f_{n}(x)\right)_{n \in \mathbb{N}}$ converges to 1 , verifies that $\left(f_{n}\right)_{n \in \mathbb{N}}$ is convergent.

It can be checked that

(i) $x$ is a smooth point of $B_{X}$ if and only if the norm of $X$ is Gâteaux differentiable at $x$; 
(ii) $x$ is a strongly smooth point of $\mathrm{B}_{X}$ if and only if the norm of $X$ is Fréchet differentiable at $x$.

It is said that a Banach space is (strongly) smooth if every point of its unit sphere is a (strongly) smooth point of its unit ball.

\section{Rotundity and smoothness}

REMARK 2.1. Let $X$ be a Banach space. We say that

(i) $X$ is strongly rotund if for every sequence $\left(x_{n}\right)_{n \in \mathbb{N}}$ in $S_{X}$ and for every $f$ in $S_{X^{*}}$. such that $\left(f\left(x_{n}\right)\right)_{n \in \mathbb{N}}$ converges to $1,\left(x_{n}\right)_{n \in \mathbb{N}}$ is convergent (see [5, pages 467-472]);

(ii) $X$ has the Efimov-Stechkin property if for every sequence $\left(x_{n}\right)_{n \in \mathbb{N}}$ in $S_{X}$ and for every $f$ in $S_{X^{*}}$ such that $\left(f\left(x_{n}\right)\right)_{n \in \mathbb{N}}$ converges to $1,\left(x_{n}\right)_{n \in \mathbb{N}}$ has a convergent subsequence (see [5, pages 478-479]);

(iii) $X$ is almost-rotund if all closed convex subsets of $S_{X}$ are compact [1].

Ivan Singer proved in 1964 (see [6]) the following:

(a) A Banach space is strongly rotund if and only if it has the Efimov-Stechkin property and is rotund.

(b) A Banach space has the Efimov-Stechkin property if and only if it is reflexive and has the Radon-Riesz property.

THEOREM 2.2. Let $X$ be a Banach space. The following assertions are equivalent:

(i) X has the Efimov-Stechkin property.

(ii) $X$ is reflexive, almost-rotund and every exposed face of $\mathrm{B}_{X}$ is a strongly exposed face of $\mathrm{B}_{X}$.

ProOF. Assume that (i) holds. It is easy to see that $X$ is reflexive and almost-rotund. Let $C$ be an exposed face of $\mathrm{B}_{X}$. Let $f \in \mathrm{S}_{X}$. be such that $C=f^{-1}(\{1\}) \cap \mathrm{B}_{X}$. Let $U$ be an open subset of $\mathrm{B}_{X}$ such that $C \subseteq U$. Suppose that there does not exist $\delta>0$ such that $\operatorname{sic}\left(\mathrm{B}_{X}, f, \delta\right) \subseteq U$. Then, for every $n \in \mathbb{N}$, there exists $x_{n} \in \operatorname{slc}\left(\mathrm{B}_{X}, f, 1 / n\right) \backslash U$. It is clear that the sequence $\left(f\left(x_{n}\right)\right)_{n \in \mathbb{N}}$ converges to 1 . Therefore, $\left(x_{n}\right)_{n \in \mathbb{N}}$ has a convergent subsequence to some element of $C$. However, the last assertion is in contradiction with the fact that $x_{n} \notin U$ for every $n \in \mathbb{N}$.

Assume that (ii) holds. Let $g \in S_{X^{*}}$ and let $\left(x_{n}\right)_{n \in \mathbb{N}}$ be a sequence in $S_{X}$ such that $\left(g\left(x_{n}\right)\right)_{n \in \mathbb{N}}$ converges to 1 . Since $g^{-1}(\{1\}) \cap \mathrm{B}_{X}$ is an exposed face of $\mathrm{B}_{X}$, there exists a functional $f$ in $S_{X}$. that characterizes it as a strongly exposed face of $\mathbf{B}_{X}$. Check that $\left(f\left(x_{n}\right)\right)_{n \in \mathbb{N}}$ converges to 1 . Since $X$ is reflexive, we can suppose, without loss of generality, that $\left(x_{n}\right)_{n \in \mathbb{N}} \omega$-converges to some element in $g^{-1}(\{1\}) \cap \mathrm{B}_{X}$. Since 
$g^{-1}(\{1\}) \cap \mathrm{B}_{X}=f^{-1}(\{1\}) \cap \mathrm{B}_{X}$, we deduce that $\left(f\left(x_{n}\right)\right)_{n \in N}$ converges to 1 . Now we will make a subsequence $\left(x_{n_{k}}\right)_{k \in \mathbb{N}}$ of $\left(x_{n}\right)_{n \in \mathbb{N}}$ such that

$$
\operatorname{dist}\left(\left\{x_{n_{k}}: k \in \mathbb{N}\right\}, f^{-1}(\{1\}) \cap B_{X}\right)=0 .
$$

For every $k \in \mathbb{N}$, let $U_{k}$ be an open subset of $\mathrm{B}_{X}$ such that $f^{-1}(\{1\}) \cap \mathrm{B}_{X} \subset U_{k}$ and $\operatorname{dist}\left(\{u\}, f^{-1}(\{1\}) \cap B_{x}\right)<1 / k$ for every $u \in U_{k}$. For every $k \in \mathbb{N}$, let $\delta_{k}$ be a positive real number such that $\operatorname{slc}\left(\mathrm{B}_{X}, f, \delta_{k}\right) \subseteq U_{k}$. For every $k \in \mathbb{N}$, let $n_{k}$ be a natural number such that $n_{k}>n_{k-1}$ and $x_{n_{k}} \in \operatorname{sic}\left(\mathrm{B}_{X}, f, \delta_{k}\right)$. The compacity of $f^{-1}(\{1\}) \cap \mathrm{B}_{X}$ allows us to deduce the result.

COROLlARY 2.3. Let $X$ be a Banach space. The following assertions are equivalent:

(i) $X$ is strongly rotund.

(ii) $X$ is reflexive and strongly exposed.

THEOREM 2.4. Let $X$ be a Banach space and let $x \in \mathrm{S}_{X}$. The following assertions are equivalent:

(i) $x$ is a rotund point of $\mathrm{B}_{X}$.

(ii) For every $y \in S_{X} \backslash\{x\}, \lim _{t \rightarrow 0^{+}}((\|x+t y\|-\|x\|) / t)<1$.

Proof. Assume that (i) holds. Let $y \in S_{X} \backslash\{x\}$. Then $\|(x+y) / 2\|<1$, therefore

$$
\frac{\|x+1 y\|-\|x\|}{1}<1 \text {. }
$$

Since the mapping

$$
\begin{aligned}
\mathbb{R} \backslash\{0\} & \longrightarrow \mathbb{R} \\
t & \longmapsto(\|x+t y\|-\|x\|) / t
\end{aligned}
$$

is increasing, we deduce that (ii) holds.

Assume that (ii) holds. Let $y \in S_{X}$ be such that $\|(x+y) / 2\|=1$. Take a functional $f \in S_{X}$. such that $\{x, y\} \subseteq f^{-1}(\{1\}) \cap B_{X}$. For every $t>0$, we have

$$
1=f(y)=\frac{f(x+t y)-f(x)}{t} \leq \frac{\|x+t y\|-\|x\|}{t} .
$$

Therefore, if we suppose that $y \neq x$, then

$$
1 \leq \lim _{t \rightarrow 0^{+}}\left(\frac{\|x+t y\|-\|x\|}{t}\right)<1
$$

which is a contradiction. 
THEOREM 2.5. Let $X$ be a Banach space. The following assertions are equivalent:

(i) $X$ is rotund.

(ii) If $C$ is a closed convex subset of $S_{X}$ such that $B_{X} \backslash C$ is convex, then $C$ is a face of $\mathrm{B}_{X}$.

Proof. It is clear that (i) implies (ii). Assume that (ii) holds and $X$ is not rotund. There exists a proper face $D$ of $\mathrm{B}_{X}$ with $\operatorname{diam}(D)>0$. Let $f \in \mathrm{S}_{X}$. be such that $f(D) \cap(0, \infty) \neq \varnothing$ and $f(D) \cap(-\infty, 0) \neq \varnothing$. Consider the set $C=D \cap$ $f^{-1}([0, \infty))$. It is clear that $C$ is closed and convex.

We check that $C$ is not a face of $\mathrm{B}_{X}$. Fix $c$ in $C$ and $d$ in $D$ with $f(c)>0$ and $f(d)<0$. Since

$$
\lim _{t \rightarrow 1^{-}}\left(\frac{1-t}{t}(-f(d))\right)=0
$$

there exists $t \in(0,1)$ such that $((1-t) / t)(-f(d))<f(c)$. We deduce that $f(t c+$ $(1-t) d)>0$. Therefore, $C$ is not a face of $B_{X}$.

Next we check that $\mathrm{B}_{X} \backslash C$ is convex. Let $x$ and $y$ be in $\mathrm{B}_{X} \backslash C$. Suppose that there exists $t \in(0,1)$ such that $t x+(1-t) y$ is in $C$. Since $D$ is a face of $\mathrm{B}_{X}$, we deduce that $x$ and $y$ are both in $D$. Since $x$ and $y$ are not in $C$, we obtain that $f(x)<0$ and $f(y)<0$. Therefore, $f(t x+(1-t) y)<0$, a contradiction with the fact that $t x+(1-t) y$ is in $C$.

LEMMA 2.6. Let $X$ be a Banach space. Let $\left(x_{n}\right)_{n \in \mathbb{N}}$ and $\left(y_{n}\right)_{n \in \mathbb{N}}$ be sequences in $\mathrm{B}_{X}$ such that $\left(\left\|\left(x_{n}+y_{n}\right) / 2\right\|\right)_{n \in \mathbb{N}}$ converges to 1 . For every $n \in \mathbb{N}$, let $\lambda_{n}$ be in $[0,1]$. Then the sequence $\left(\left\|\lambda_{n} x_{n}+\left(1-\lambda_{n}\right) y_{n}\right\|\right)_{n \in \mathbb{N}}$ is convergent to 1 .

PROOF. We assume, without loss of generality, that $\left(\lambda_{n}\right)_{n \in \mathbb{N}}$ is convergent to some $\lambda$ in $[0,1]$. It will be enough to prove that the sequence $\left(\left\|\lambda x_{n}+(1-\lambda) y_{n}\right\|\right)_{n \in \mathbb{N}}$ is convergent to 1 . Assume that $\lambda$ is in $[0,1 / 2]$. If $\lambda$ is in $[1 / 2,1]$, then we can reason similarly. For every $n \in \mathbb{N}$, set $z_{\lambda}^{n}=\lambda x_{n}+(1-\lambda) y_{n}$. Then

$$
\begin{aligned}
\left\|\frac{x_{n}+y_{n}}{2}\right\| & =\left\|\frac{1}{2} x_{n}+\frac{1}{2}\left(\frac{1}{1-\lambda} z_{\lambda}^{n}-\frac{\lambda}{1-\lambda} x_{n}\right)\right\|=\left\|\frac{1-2 \lambda}{2-2 \lambda} x_{n}+\frac{1}{2-2 \lambda} z_{\lambda}^{n}\right\| \\
& \leq \frac{1-2 \lambda}{2-2 \lambda}\left\|x_{n}\right\|+\frac{1}{2-2 \lambda}\left\|z_{\lambda}^{n}\right\| \leq \frac{1-2 \lambda}{2-2 \lambda}+\frac{1}{2-2 \lambda}\left\|z_{\lambda}^{n}\right\| \\
& \leq \frac{1-2 \lambda}{2-2 \lambda}+\frac{1}{2-2 \lambda}=1 .
\end{aligned}
$$

Therefore, the sequence

$$
\left(\frac{1-2 \lambda}{2-2 \lambda}+\frac{1}{2-2 \lambda}\left\|z_{\lambda}^{n}\right\|\right)_{n \in \mathbb{N}}
$$


converges to 1 . So, the sequence $\left(\left\|z_{\lambda}^{n}\right\|\right)_{n \in \mathbb{N}}$ converges to 1 .

THEOREM 2.7. Let $X$ be a Banach space and let $x \in \mathrm{S}_{X}$. The following assertions are equivalent:

(i) $x$ is a locally uniformly rotund point of $\mathrm{B}_{X}$.

(ii) If $\left(C_{n}\right)_{n \in \mathbb{N}}$ is a sequence of convex subsets of $\mathrm{B}_{X}$ such that the sequence $\left(\operatorname{dist}\left(\{0\}, C_{n}\right)\right)_{n \in \mathbb{N}}$ converges to 1 , and $x$ is a diametral point of $C_{n}$ for every $n \in \mathbb{N}$, then the sequence $\left(\operatorname{diam}\left(C_{n}\right)\right)_{n \in \mathbb{N}}$ converges to 0 .

PROOF. Assume that (i) holds. Let $\left(C_{n}\right)_{n \in \mathbb{N}}$ be a sequence of convex subsets of $\mathrm{B}_{X}$ such that the sequence (dist $\left.\left(\{0\}, C_{n}\right)\right)_{n \in \mathbb{N}}$ converges to 1 and, for every $n \in \mathbb{N}, x$ is a diametral point of $C_{n}$. Since, for every $n \in \mathbb{N}, \operatorname{diam}\left(C_{n}\right)=\sup \left(\left\{\|x-c\|: c \in C_{n}\right\}\right)$, there exists $y_{n} \in C_{n}$ such that $\operatorname{diam}\left(C_{n}\right)<\left\|x-y_{n}\right\|+1 / n$. Since $\left(x+y_{n}\right) / 2$ is in $C_{n}$, we deduce that

$$
\operatorname{dist}\left(\{0\}, C_{n}\right) \leq\left\|\frac{x+y_{n}}{2}\right\| \leq 1
$$

Since $x$ is a locally uniformly rotund point of $\mathrm{B}_{X}$, we obtain that $\left(\operatorname{diam}\left(C_{n}\right)\right)_{n \in \mathbb{N}}$ converges to 0 .

Assume that (ii) holds. Let $\left(y_{n}\right)_{n \in \mathbb{N}}$ be a sequence in $S_{X}$ such that the sequence $\left(\left\|\left(x+y_{n}\right) / 2\right\|\right)_{n \in \mathbb{N}}$ converges to 1 . For every $n \in \mathbb{N}$, let $C_{n}$ be the segment of extremes $y_{n}$ and $x$. It is clear that $x$ is a diametral point of $C_{n}$. In addition, according to Lemma 2.6, it is not difficult to see that $\left(\operatorname{dist}\left(\{0\}, C_{n}\right)\right)_{n \in \mathbb{N}}$ converges to 1 . Therefore, the sequence $\left(\operatorname{diam}\left(C_{n}\right)\right)_{n \in \mathbb{N}}$ converges to 0 . In consequence, $\left(y_{n}\right)_{n \in \mathbb{N}}$ converges to $x$.

REMARK 2.8. It is known that a Banach space is uniformly rotund (see [5, pages 441459]) if and only if for every pair of sequences $\left(x_{n}\right)_{n \in \mathbb{N}}$ and $\left(y_{n}\right)_{n \in \mathbb{N}}$ in $S_{X}$ such that $\left(\left\|\left(x_{n}+y_{n}\right) / 2\right\|\right)_{n \in \mathbb{N}}$ converges to $1,\left(x_{n}-y_{n}\right)_{n \in \mathbb{N}}$ converges to 0 . It is also well known (see [5, page 460]) that every uniformly rotund Banach space is a locally uniformly rotund Banach space. Moreover, this implication is not an equivalence (see [5, pages 462-463]).

A little modification of proof of Theorem 2.7 yields the equivalence of these assertions for a Banach space $X$ :

(a) $X$ is uniformly rotund.

(b) If $\left(C_{n}\right)_{n \in \mathbb{N}}$ is a sequence of convex subsets of $\mathrm{B}_{X}$ such that the sequence $\left(\operatorname{dist}\left(\{0\}, C_{n}\right)\right)_{n \in \mathbb{N}}$ converges to 1 , then the sequence $\left(\operatorname{diam}\left(C_{n}\right)\right)_{n \in \mathbb{N}}$ converges to 0 .

REMARK 2.9. Recently, Bandyopadhyay and Lin [3] proved the following equivalences for a point $x$ of the unit sphere of a Banach space $X$ : 
(i) $x$ is a rotund point of $B_{X}$ if and only if it is an exposed point of $B_{X}$ for each $f \in \mathrm{S}_{X^{*}}$ such that $f(x)=1$.

(ii) $x$ is an almost locally uniformly rotund point of $\mathrm{B}_{X}$ if and only if it is a strongly exposed point of $\mathrm{B}_{X}$ for each $f \in \mathrm{S}_{X^{*}}$ such that $f(x)=1$.

According to these results, it is clear that

(i) if $x$ is an exposed point and a smooth point of $B_{X}$, then it is a rotund point of $\mathrm{B}_{X}$;

(ii) if $x$ is a strongly exposed point and a smooth point of $\mathbf{B}_{X}$, then it is an almost locally uniformly rotund point of $\mathrm{B}_{X}$.

THEOREM 2.10. Let $X$ be a Banach space and let $x \in \mathrm{S}_{X}$. If $x$ is a strongly exposed point of $\mathrm{B}_{X}$ and a strongly smooth point of $\mathrm{B}_{X}$, then it is a locally uniformly rotund point of $\mathrm{B}_{X}$.

PROOF. Let $\left(y_{n}\right)_{n \in \mathbb{N}}$ be a sequence in $S_{X}$ such that $\left(\left\|\left(x+y_{n}\right) / 2\right\|\right)_{n \in \mathbb{N}}$ converges to 1 . Take the functional $f$ in $S_{X}$. that characterizes $x$ as a strongly exposed point of $\mathrm{B}_{X}$. For every $n \in \mathbb{N}$, let $f_{n}$ in $\mathrm{S}_{X^{*}}$ be such that

$$
f_{n}\left(\frac{x+y_{n}}{2}\right)=\left\|\frac{x+y_{n}}{2}\right\| \text {. }
$$

Since $\left(f_{n}\left(\left(x+y_{n}\right) / 2\right)\right)_{n \in \mathbb{N}}$ converges to 1 , we assume, without loss of generality, that the sequences $\left(f_{n}\left(y_{n}\right)\right)_{n \in \mathbb{N}}$ and $\left(f_{n}(x)\right)_{n \in \mathbb{N}}$ are both convergent to 1 . Since $x$ is a strongly smooth point of $\mathrm{B}_{X}$, we deduce that $\left(f_{n}\right)_{n \in \mathbb{N}}$ converges to $f$. For every $n \in \mathbb{N}$, we have that

$$
\left|1-f\left(y_{n}\right)\right| \leq\left|1-f_{n}\left(y_{n}\right)\right|+\left|f_{n}\left(y_{n}\right)+f\left(y_{n}\right)\right| \leq\left|1-f_{n}\left(y_{n}\right)\right|+\left\|f_{n}-f\right\| .
$$

Therefore, $\left(f\left(y_{n}\right)\right)_{n \in \mathbb{N}}$ converges to 1 . Since $x$ is a strongly exposed point of $\mathrm{B}_{X}$, we deduce that the sequence $\left(y_{n}\right)_{n \in \mathbb{N}}$ converges to $x$.

COROLlARY 2.11. Let $X$ be a Banach space. Then $X$ is locally uniformly rotund if it is almost locally uniformly rotund and its norm is Fréchet differentiable in $S_{X}$.

\section{Renormings}

REMARK 3.1. Let $X$ be a Banach space. Let $f$ be a norm-attaining functional of $S_{X}$. The mapping $\|\cdot\|_{0}: X \rightarrow \mathbb{R}$, defined for every $y$ in $X$ by the formula

$$
\|y\|_{0}=\frac{1}{\sqrt{2}} \sqrt{\|y\|^{2}+|f(y)|^{2}}
$$

is an equivalent norm on $X$ such that (where $X_{0}$ denotes the space $X$ with the norm $\left.\|\cdot\|_{0}\right)$ 
(i) $\mathrm{B}_{X} \subseteq \mathrm{B}_{X_{0}} \subseteq \sqrt{2} \mathrm{~B}_{X}$;

(ii) $\mathrm{S}_{X_{0}} \cap \mathrm{S}_{X}=C \cup-C$, where $C=f^{-1}(\{1\}) \cap \mathrm{B}_{X}$;

(iii) $f$ is in $S_{X_{0}^{*}}$.

THEOREM 3.2. Let $X$ be a Banach space. Let $C$ be a nonempty subset of $\mathrm{S}_{X}$. The following statements are equivalent:

(i) $C$ is an exposed face of $\mathrm{B}_{X}$.

(ii) There exists an equivalent norm $\|\cdot\|_{0}$ on $X$ such that $\mathrm{B}_{X} \subseteq \mathrm{B}_{X_{0}} \subseteq \sqrt{2} \mathrm{~B}_{X}$, $\mathrm{S}_{X_{0}} \cap \mathrm{S}_{X}=C \cup-C$, and $C$ is a maximal face of $\mathrm{B}_{X_{0}}$, where $X_{0}$ denotes the space $X$ with the norm $\|\cdot\|_{0}$.

ProOF. It is an easy exercise to see that (ii) implies (i). Assume that (i) holds. Let $f \in \mathrm{S}_{X^{*}}$ be the functional that characterizes $C$ as an exposed face of $\mathrm{B}_{X}$. Consider the equivalent norm on $X$ given by (1). We know that $B_{X} \subseteq B_{X_{0}} \subseteq \sqrt{2} B_{X}$ and $\mathrm{S}_{X_{0}} \cap \mathrm{S}_{X}=C \cup-C$. We see that $C$ is a maximal face of $\mathrm{B}_{X_{0}}$. Let $D$ be a face of $\mathrm{B}_{X_{0}}$ such that $C \subset D$ and consider an element $d$ in $D \backslash C$. Since $\|d\|_{0}=1$, we have that $\|d\|^{2}+|f(d)|^{2}=2$, therefore $\|d\|+|f(d)| \leq 2$. Since $\|d\|>1$ (because $d$ is not in $C \cup-C)$, we deduce that $|f(d)|<1$. Therefore, $\|d\|+|f(d)|<2$. Let $c$ be in $C$. Let us see that $\|(c+d) / 2\|_{0}<1$, which is a contradiction. We have that

$$
\begin{aligned}
\left\|\frac{c+d}{2}\right\|_{0} & =\frac{1}{\sqrt{2}} \sqrt{\left\|\frac{c+d}{2}\right\|^{2}+\left|f\left(\frac{c+d}{2}\right)\right|^{2}} \\
& =\frac{1}{2 \sqrt{2}} \sqrt{\|c+d\|^{2}+|1+f(d)|^{2}} \\
& \leq \frac{1}{2 \sqrt{2}} \sqrt{\|c\|^{2}+\|d\|^{2}+2\|c\|\|d\|+1+|f(d)|^{2}+2|f(d)|} \\
& =\frac{1}{2 \sqrt{2}} \sqrt{4+2(\|d\|+|f(d)|)}=\frac{1}{2} \sqrt{2+\|d\|+|f(d)|}<1 .
\end{aligned}
$$

So the proof is concluded.

COROLLARY 3.3. Let $X$ be a Banach space and let $x \in \mathrm{S}_{X}$. The following statements are equivalent:

(i) $x$ is an exposed point of $\mathrm{B}_{X}$.

(ii) There exists an equivalent norm $\|\cdot\|_{0}$ on $X$ such that $\mathrm{B}_{X} \subseteq \mathrm{B}_{X_{0}} \subseteq \sqrt{2} \mathrm{~B}_{X}$, $\mathrm{S}_{X_{0}} \cap \mathrm{S}_{X}=\{x,-x\}$, and $x$ is a rotund point of $\mathrm{B}_{X_{0}}$, where $X_{0}$ denotes the space $X$ with the norm $\|\cdot\|_{0}$.

THEOREM 3.4. Let $X$ be a Banach space and let $x \in \mathrm{S}_{X}$. The following statements are equivalent: 
(i) $x$ is a strongly exposed point of $\mathrm{B}_{\mathrm{X}}$.

(ii) There exists an equivalent norm $\|\cdot\|_{0}$ on $X$ such that $\mathrm{B}_{X} \subseteq \mathrm{B}_{X_{0}} \subseteq \sqrt{2} \mathrm{~B}_{X}$, $S_{X_{0}} \cap S_{X}=\{x,-x\}$, and $x$ is a locally uniformly rotund point of $B_{X_{0}}$, where $X_{0}$ denotes the space $X$ with the norm $\|\cdot\|_{0}$.

PROOF. It is an easy exercise to see that (ii) implies (i). Assume that (i) holds. Let $f \in \mathrm{S}_{X^{*}}$ be the functional that characterizes $x$ as a strongly exposed point of $\mathrm{B}_{X}$. Consider the equivalent norm on $X$ given by (1). We know that $\mathrm{B}_{X} \subseteq \mathrm{B}_{X_{0}} \subseteq \sqrt{2} \mathrm{~B}_{X}$ and $S_{X_{0}} \cap S_{X}=\{x,-x\}$. We show that $x$ is a locally uniformly rotund point of $B_{X_{0}}$. Let $\left(x_{n}\right)_{n \in \mathrm{N}}$ be a sequence in $S_{X_{0}}$ such that the sequence

$$
\left(\left\|\frac{x_{n}+x}{2}\right\|_{0}\right)_{n \in \mathbb{N}}
$$

converges to 1. We assume, without loss of generality, that there exist $\alpha$ and $\beta$ in $\mathbb{R}$ such that $\left(\left\|x_{n}\right\|\right)_{n \in \mathbb{N}}$ converges to $\alpha$ and $\left(f\left(x_{n}\right)\right)_{n \in \mathbb{N}}$ converges to $\beta$. For every $n \in \mathbb{N}$, since $\left\|x_{n}\right\|_{0}=1$, we have that $\left\|x_{n}\right\|^{2}+\left|f\left(x_{n}\right)\right|^{2}=2$ and $\left\|x_{n}\right\|+\left|f\left(x_{n}\right)\right| \leq 2$. On the other hand, for every $n \in \mathbb{N}$, we have

$$
\begin{aligned}
\left\|\frac{x_{n}+x}{2}\right\|_{0} & =\frac{1}{\sqrt{2}} \sqrt{\left\|\frac{x_{n}+x}{2}\right\|^{2}+\left|f\left(\frac{x_{n}+x}{2}\right)\right|^{2}} \\
& =\frac{1}{2 \sqrt{2}} \sqrt{\left\|x_{n}+x\right\|^{2}+\left|f\left(x_{n}\right)+1\right|^{2}} \\
& \leq \frac{1}{2 \sqrt{2}} \sqrt{\left\|x_{n}\right\|^{2}+\|x\|^{2}+2\left\|x_{n}\right\|\|x\|+1+\left|f\left(x_{n}\right)\right|^{2}+2\left|f\left(x_{n}\right)\right|} \\
& =\frac{1}{2 \sqrt{2}} \sqrt{4+2\left(\left\|x_{n}\right\|+\left|f\left(x_{n}\right)\right|\right)}=\frac{1}{2} \sqrt{2+\left\|x_{n}\right\|+\left|f\left(x_{n}\right)\right|} \leq 1 .
\end{aligned}
$$

Therefore,

$$
1 \leq \frac{1}{2} \sqrt{2+\alpha+|\beta|} \leq 1 .
$$

We deduce that $\alpha=|\beta|=1$. Suppose that $\beta=-1$. Then $\left((-f)\left(x_{n}\right)\right)_{n \in \mathbb{N}}$ converges to 1 , therefore $\left(x_{n}\right)_{n \in \mathbb{N}}\|\cdot\|$-converges to $-x$. Then, for every $n \in \mathbb{N}$, we have

$$
\left\|x_{n}+x\right\|_{0}=\sqrt{\left\|x_{n}+x\right\|^{2}+\left|f\left(x_{n}+x\right)\right|^{2}} .
$$

Therefore, $\left(\left\|x_{n}+x\right\|_{0}\right)_{n \in \mathbb{N}}$ converges to 0 . We have a contradiction, because $\left(\|\left(x_{n}+\right.\right.$ $\left.x) / 2 \|_{0}\right)_{n \in \mathbb{N}}$ converges to 1 . We deduce that $\alpha=\beta=1$. Then, $\left(f\left(x_{n}\right)\right)_{n \in \mathbb{N}}$ converges to 1 , therefore $\left(x_{n}\right)_{n \in \mathbb{N}}\|\cdot\|$-converges to $x$. Then, for every $n \in \mathbb{N}$, we have

$$
\left\|x_{n}-x\right\|_{0}=\sqrt{\left\|x_{n}-x\right\|^{2}+\left|f\left(x_{n}-x\right)\right|^{2}} .
$$

Therefore, $\left(x_{n}\right)_{n \in \mathbb{N}}\|\cdot\|_{0}$-converges to $x$. 


\section{References}

[1] J. Banas, 'On drop property and nearly uniformly smooth Banach spaces', Nonlinear Anal. 14 (1990), 927-933.

[2] P. Bandyopadhyay, D. Huang, B.-L. Lin and S. L. Troyanski, 'Some generalizations of locally uniform rotundity', J. Math. Anal. Appl. 252 (2000), 906-916.

[3] P. Bandyopadhyay and B.-L. Lin, 'Some properties related to nested sequence of balls in Banach spaces', Taiwanese J. Math. 5 (2001), 19-34.

[4] J. R. Giles, 'Strong differentiability of the norm and rotundity of the dual', J. Austral. Math. Soc. 26 (1978), 302-308.

[5] R. E. Megginson, An introduction to Banach space theory (Springer, New York, 1998).

[6] I. Singer, 'Some remarks on approximative compactness', Rev. Roumaine Math. Pures Appl. 9 (1964), 167-177.

Departamento de Matemáticas

Facultad de Ciencias

Universidad de Cádiz

Polígono Río San Pedro

11.510 Puerto Real (Cádiz)

Spain

e-mail: antonio.aizpuru@uca.es, fjavier.garcia@uca.es 